\title{
El Desafío de Evaluar por Competencias en la Universidad. Reflexiones y Experiencias Prácticas
}

\section{The Challenge of Evaluating by Competences in Higher Education. Reflections and Practical Experiences}

\author{
Miguel A. Zabalza Beraza * 1 \\ Laura Lodeiro Enjo ${ }^{2}$ \\ ${ }^{1}$ Universidad de Santiago de Compostela, España \\ ${ }^{2}$ Pontificia Universidad Católica Madre y Maestra, República Dominicana
}

\begin{abstract}
El objetivo de este artículo es ofrecer una reflexión sobre la evaluación por competencias en la educación superior. Consta de dos partes. En la primera, se analiza el sentido y las condiciones didácticas aplicables a cada uno de los tres conceptos centrales que organizan esta aportación (evaluación, competencia y evaluación de competencias). En la segunda, se presentan dos experiencias concretas de evaluación por competencias en nuestras universidades: una en la que las competencias se vinculan a las diversas materias del plan de estudios; otra en la que la evaluación de competencias aparece al final de la carrera como expresión de la formación global recibida: los ECOEs de las Facultades de Medicina.
\end{abstract}

Palabras clave: Educación superior; Evaluación; Competencias; Evaluación por competencias; Enseñanza universitaria.

The purpose of this article is to reflect about the assessment by competences at the university. It consists of two parts. In the first one, the meaning and didactic conditions applicable to each of the three central concepts that organize our contribution (assessment, competence and assessment by competences) are analyzed. In the second part, two concrete experiences of evaluation by competences in our universities are presented: one in which competences are linked to the various subjects of the curriculum; another one in which the assessment of the competences appears at the end of the career as an expression of the global training received: the ECOEs of the Faculties of Medicine.

Keywords: Higher education; Evaluation; Competencies; Competencies-based evaluation; Teaching at university.

*Contacto: miguel.zabalza@usc.es

issn: 1989-0397

www.rinace.net/riee/

https://revistas.uam.es/riee
Recibido: 4 de mayo de 2019

$1^{\text {a }}$ Evaluación: 3 de septiembre de 2019

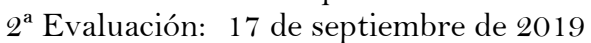

Aceptado: $\quad 24$ de septiembre de 2019 


\section{Introducción}

El objetivo de esta aportación es ofrecer una reflexión sobre la evaluación de competencias en la universidad enriquecida con experiencias reales. Se estructura en dos partes: En la primera (compuesta por los tres primeros epígrafes) se exponen algunas condiciones básicas que debe reunir la evaluación en las instituciones de educación superior, se plantean algunas reglas y condiciones del enfoque por competencias y, siguiendo la misma lógica, las condiciones de la evaluación por competencias. En la segunda parte, con el propósito de mostrar ejemplos, se presentan dos experiencias sobre cómo en dos contex tos diferentes se ha abordado el enfoque por competencias y la evaluación de las mismas.

La primera de las experiencias se desarrolla en la Pontificia Universidad Católica Madre y Maestra (PUCMM), una universidad de República Dominica que, tras la creación de su nuevo modelo educativo basado en el enfoque por competencias en 2011, ha abocado a la institución a afrontar el rediseño de toda su oferta desde la nueva perspectiva. Se realizó un pequeño estudio exploratorio, a través de la realización de entrevistas en profundidad a profesores de la Institución, con el propósito de hacer una primera aproximación a las impresiones que tienen con respecto al desarrollo de las asignaturas por competencias y la evaluación de estas tras los primeros años de implementación. Tanto el guion de la entrevista como el análisis se estructuran en función de las premisas expuestas en la primera parte del artículo. Pese a que aún no se ha completado todo el proceso de cambio, el camino seguido nos permite indagar sobre el proceso de cambio mental y operativo que los docentes van realizando en la implantación del nuevo enfoque tanto en lo que se refiere a la docencia en general como, más específicamente, en relación a la evaluación.

La segunda experiencia parte de postulados diferentes. Se conciben las competencias como unidades curriculares de carácter molar y transversal. Una especie de resumen integrado del conocimiento profesional que el programa de estudios postula. Por ese motivo se analiza el caso de las ECOEs (Evaluación Clínica Objetiva Estructurada) de las Facultades de Medicina donde se entienden las competencias como síntesis final de la formación recibida. Frente a la tendencia general a vincular las competencias a las disciplinas individuales, las ECOEs buscan situaciones más globales y, por tanto, analizan performances que requieren el dominio de situaciones complejas y la integración de aprendizajes provenientes de diferentes disciplinas

\section{Evaluar en la universidad}

La importancia de la evaluación en los sistemas formativos universitarios es obvia. Probablemente porque es el componente curricular del que se derivan repercusiones más dramáticas para los estudiantes. Algunas de esas consecuencias son visibles y objetivables: las repercusiones académico-administrativas (si aprueban o no, si promocionan o no, si obtienen el título o no, si logran mantener un expediente académico de "excelencia" o se han de conformar con uno mediocre) o las económicas (pagar nueva matrícula, tener que mantenerse fuera de casa por más tiempo, no poder acceder a una beca, etc.). También hay consecuencias poco tangibles: la forma en que la evaluación repercute en su moral y su autoestima, la forma en que afecta a su motivación hacia el aprendizaje, las repercusiones familiares sobrevenidas, entre otras.

Por lo que se refiere a los docentes, la evaluación constituye un espacio saturado de dilemas. Salvo excepciones, la evaluación genera más preocupación que satisfacciones. Por 
lo general, disfrutamos bastante de las clases, pero esa satisfacción se frustra cuando tenemos que evaluar porque se quiebra el marco relacional constructivo que la docencia permite, pero la evaluación dificulta. Nuestro papel amable de facilitadores y guías se ve complicado por el de juzgadores.

En definitiva, la aproximación que los docentes hacemos a la evaluación es variada e idiosincrásica (situación coherente con el principio de la libertad de cátedra que para algunos no es otra cosa que la desregulación del quehacer docente). Va desde los aprobados generales a los reprobados masivos; desde los exámenes finales y únicos al permanente estado de evaluación en el que todo cuenta; desde las evaluaciones sorpresa a las negociadas; de las presenciales y con lugares fijados por los docentes, a las virtuales y realizadas desde casa. Un panorama polícromo y muy vinculado a lo que cada docente o cada tradición académica entiende como evaluación pertinente.

En ese marco y antes de pasar a hablar de evaluación por competencias, nos gustaría dejar claras algunas condiciones básicas que debe reunir la evaluación en la universidad.

\subsection{La primera condición es considerar que evaluación forma parte del currículo universitario}

Es decir, forma parte del proyecto formativo (eso es el currículo) que cada Facultad desarrolla (Zabalza y Zabalza Cerdeiriña, 2018. Y no lo hace como un elemento secundario de dicho proyecto sino, más bien, como su estrella polar, la que marca el camino de lo importante, la que orienta a estudiantes y profesores en el camino a seguir y monitoriza sus avances.

\subsection{La segunda condición tiene que ver con la naturaleza bifronte de la evaluación universitaria}

Además de la cara formativa propia de las evaluaciones en cualquier nivel educativo (Conan,1999), evaluar en la universidad incluye la condición de la acreditación. Se supone que las evaluaciones en sede universitaria no solo hablan del esfuerzo y mérito del estudiante, sino que deben acreditar su competencia profesional en el ámbito al que la evaluación aluda. La suma de las acreditaciones parciales en las diversas asignaturas o la evaluación global que al finalizar sus estudios se emita, es lo que da derecho a obtener un título profesional y, por tanto, garantiza que esa persona es competente, al menos en grado básico, para poder desempeñar las tareas que como profesional se le encomendarán. Esa es la garantía que se espera de las instituciones universitarias y la condición básica para su propia acreditación (véanse los criterios de la European Consortium for Accreditation in Higher education, ECA, 2013).

\subsection{Una tercera condición alude a la exigencia de objetividad en la evaluación}

Una evaluación nunca puede ser plenamente objetiva pues siempre incluye una valoración por parte del evaluador, pero ha de acercarse lo más posible a esa condición. Y eso, no solo porque se afecta a derechos individuales de los estudiantes, sino porque es una exigencia intrínseca a toda evaluación. Evaluar no es conocer algo, ni opinar sobre algo. Evaluar es un proceso complejo que tiene sus reglas y condiciones y que, por tanto, queda lejos de un mero conocimiento incidental, de una simple intuición o de la expresión de una opinión. Evaluar significa "emitir un juicio de valor sobre algo, basándose en datos fehacientes y sistemáticos". Incluye, por tanto, la obtención de evidencias objetivas (o lo más cercanas a la objetividad que sea posible) sobre las que se emitirá un juicio de valor. Ni siquiera la 
valoración que podamos emitir sobre las evidencias obtenidas está libre de esta exigencia de objetividad y transparencia. Valorar, en este caso, significa comparar dichas evidencias con un parámetro que nos sirva de referencia, con un normotipo. Según sea dicho normotipo podremos hacer evaluaciones referidas a criterio, referidas a la norma estadística, o personalizadas. En cualquier caso, ese marco de referencia debe estar bien explicitado y debe ser transparente. Pese a todo ello, la evaluación siempre tendrá su residuo de subjetividad porque siempre incluirá un juicio de valor. La cuestión está en minimizar sus efectos, en evitar la incertidumbre que generan las evaluaciones muy dependientes del contexto y/o del evaluador de turno.

\subsection{La evaluación se concibe, a veces, como la fase final de un proceso formativo}

Evaluar para constatar si hemos alcanzado las metas previstas) pero su función curricular es más potente, si cabe, como componente transversal de todo el proceso formativo y como nexo de conexión y continuidad entre fases consecutivas de la formación. La estructura clásica de un proceso formativo (planear, ejecutar, evaluar, ajustar) sitúa a la evaluación como elemento básico de todos ellos. Cuando Oser y Baeriswyl (2001) describen las coreografías didácticas definen la primera fase como "anticipación" (la forma en que los docentes visualizan el resultado al que desearían llegar, esto es, todo el conjunto de aprendizajes que sus estudiantes deberían alcanzar al finalizar su periodo de trabajo con ellos; es decir, lo que deberán demostrar en su evaluación final). Lo mismo hacen los planes de estudio o los programas de las disciplinas: señalar desde el inicio qué competencias se espera que los estudiantes alcancen, lo que es tanto como describir sobre qué serán evaluados para recibir su acreditación. Y la presencia de la evaluación se mantiene en la fase de ejecución de la formación y al final de la misma, como elemento de supervisión y control de la marcha del proceso. Y volverá la evaluación a adquirir todo su protagonismo en la fase de reajuste para conectar y dar continuidad al próximo periodo formativo en función de los resultados alcanzados en el anterior.

\subsection{Finalmente, la evaluación, en sí misma, forma parte de las competencias profesionales que los docentes deberíamos poseer (Houston, 1985; Zabalza, 2003)}

Como tal competencia está sujeta a las variaciones y exigencias (de progresión, actualización y perfeccionamiento) de toda competencia profesional. La pedagogía universitaria va incorporando nuevas ideas y normas sobre la naturaleza y función de la evaluación en las políticas universitarias; novedades técnicas vinculadas a las TIC y a los nuevos entornos de aprendizaje (Rodríguez-Gómez e Ibarra, 2011); nuevos enfoques y recursos para realizarla; reajustes en el rol de estudiantes y profesores a la hora de diseñar e implementar las evaluaciones (Fletcher, 1997), etc. Los docentes necesitamos un claro aggiornamento en nuestras prácticas evaluadoras (Brown y Glasner, 2003).

\section{Las competencias en el contexto académico universitario}

Es difícil saber si, a nivel global, el modelo de formación por competencias ha sido un éxito o un fracaso. Depende de a quién se le pregunte. Lo cierto es que no ha logrado el nivel de transformación de la enseñanza que tantos prometían cuando esta propuesta formativa comenzó. En definitiva, no podemos saber si, al final, el modelo de enseñanza basada en competencias acabará afianzándose o si, como ha pasado con otros enfoques formativos de 
moda durante algún tiempo, se diluirá en la nebulosa de escepticismos y prejuicios que acompañan toda innovación. Es difícil no ya predecir el futuro sino incluso saber cuál es el presente en este posmodernismo líquido que caracteriza lo educativo. Pero, suceda lo que suceda, creemos que ha sido muy interesante este paso por las competencias (y por los procesos vinculados a ellas) porque nos han abierto los ojos a condiciones y urgencias docentes a las que habitualmente prestábamos poca atención (Cano, 2008). Entre ellas, la de cómo evaluarlas, a lo que nos referiremos luego.

Sin desconsiderar otros planteamientos más críticos sobre la naturaleza de las competencias y sobre sus efectos deletéreos en la docencia (Hager, Gonczi \& Athanasou, 1994), nuestra postura es ver los nuevos enfoques basados en competencias como el fruto de la evolución del pensamiento pedagógico en su intento de resolver algunos de los problemas que la formación, y en especial la formación universitaria, nos venía planteando. Más en concreto, el problema de la articulación entre teoría y práctica, el de la profesionalización, el de la transversalidad, entre otros. Era habitual escuchar que la escuela (y la universidad) eran muy teóricas, que transmitían un conocimiento alejado de la vida y de las nuevas demandas de las profesiones, que necesitábamos una auténtica reforma curricular y de enfoque que conectara de forma más directa la formación y el mundo real de nuestro momento. También que la universidad, más allá de la especificidad de cada disciplina, no podía sustraerse al compromiso de formar a sus estudiantes en aspectos básicos como la expresión oral o escrita, la informática, los idiomas, el trabajo en equipo, la reflexión, el rigor científico, etc. Y aparecieron las competencias con sus ventajas e inconvenientes. Y, desde luego, con la pretensión de transformar muchas de las cosas que se venían haciendo en Educación Superior.

En cualquier caso, trabajar (formar) por competencias tiene sus reglas porque las competencias constituyen una forma muy particular de entender y desarrollar los procesos formativos. Esas reglas y condiciones del enfoque por competencias son las que nos gustaría aclarar en este apartado.

\subsection{Las competencias son unidades formativas molares frente a las disciplinas cuya naturaleza curricular es más concreta y específica}

Trabajar por competencias requiere una organización curricular muy reticular con núcleos transdisciplinares que permitan construir procesos formativos amplios $\mathrm{y}$ progresivos. Uno de los problemas que han afectado al desarrollo del enfoque por competencias es que se ha pretendido introducir a la fuerza en un modelo docente que es absolutamente disciplinar. No es que sea imposible esa conjunción de disciplinas y competencias, ese unir lo general con lo particular (de hecho, la experiencia que se recoge en este texto pertenece, en buena parte, a ese formato de diseño curricular) pero plantea muchas dificultades especialmente en lo que se refiere a las competencias generales. Las competencias requieren un contexto formativo global o, cuando menos, amplio (interdisciplinar) y progresivo (Yániz, 2008). El marco que generan las disciplinas aisladas resulta restrictivo y da escaso juego, sobre todo porque ayuda poco a modificar el modelo de docencia atomizada y discontinua en la que estamos acostumbrados a trabajar.

\subsection{Cuando hablamos de competencias no estamos hablando de una realidad homogénea y bien delimitada}

Las competencias son muy diferentes entre sí en su naturaleza, en su impacto sobre la formación, en su presencia real en las prácticas docentes. Houston (1985), antes de todo el 
debate que se ha producido en la década pasada, hablaba de 5 tipos de competencias (se refería a la formación de docentes): las basadas en lo cognitivo (cognitive based competencies); las basadas en las actuaciones prácticas (performance-based competencies); las competencias vinculadas a capacidades generales de los sujetos (competencia lingüística, por ejemplo); las competencias vinculadas a rasgos actitudinales (affective competencies); competencias vinculadas a las experiencias vividas (exploratory competencies). En general, la literatura especializada en competencias las ha descrito como conformadas por una estructura de tres componentes: conocimiento + habilidades + actitudes. En esa estructura canónica (siempre están presentes los tres componentes en las competencias) el peso de cada uno de esos componentes varía de unas competencias a otras. Todas incluyen los tres componentes, pero pueden hacerlo con proporciones diversas: algunas competencias saturan mucho el apartado de conocimientos con menor peso de las habilidades y actitudes (ser competente en arte gótico); en otras tiene mayor peso las habilidades que los conocimientos y actitudes (ser un chef especialista en postres, ser competente en desarrollo de software); también las hay en las que son las actitudes, más que los conocimientos o habilidades las que van a marcar la competencia (un profesional de la mediación; alguien que trabaja en un contexto de grupos marginales).

Con todo y siendo eso así, precisamos considerar que la universidad es un contexto en el cual la dimensión conocimiento tiende a priorizarse en las competencias (Sanz de Acevedo, 2016). Otras modalidades formativas pueden resultar más prácticas y centrarse en el aspecto ejecutivo de las competencias (la formación profesional, por ejemplo, o ciertos talleres de formación continuada), pero lo específico de la formación universitaria es, justamente, el enriquecimiento cognitivo de las competencias. Alguien puede llegar a ser experto en cocina, pero si su formación se hace universitaria quizás no mejore sus habilidades prácticas pero, con seguridad, deberá mejorar sus conocimientos tanto básicos (química, biología) como específicos (nutrición, conservación alimentos, etc.).

\subsection{La estructura de la formación por competencias diferencia entre competencias de diferente nivel y con diverso contenido en la malla curricular}

Hay competencias (básicas) que son propedéuticas, esto es, sirven de base a otras competencias: hace falta tener buenas bases en Biología para poder abordar ciertos conocimientos médicos; hay competencias genéricas necesarias para poder abordar otras más especializadas: dominar las matemáticas para cursar una ingeniería; hay competencias transversales que constituyen una especie de común denominador de todas las competencias del proceso formativo: saber planificar, por ejemplo, en las profesiones que implican intervenir o desarrollar proyectos; hay competencias formativas o soft competencies (Villardón-Gallego, 2015): expresión oral y escrita, dominio informático, capacidad de trato con la gente, emprendimiento, pensamiento creativo, reflexión y capacidad crítica, etc.

\subsection{Las competencias constituyen espacios amplios y flexibles de dominio conceptual, operativo y comportamental}

No se trata de realidades estancas y fijas. Este aspecto resulta esencial cuando estamos hablando de enseñanza y formación. Como señala Paricio (2019) "las competencias son como un trayecto infinito. Los aprendizajes, como un lugar específico de ese trayecto, el nivel específico de dominio de la competencia para ese tramo de la formación" (s/p). Ser competente no es ni indica un estado o una cualidad delimitada; se es competente (o no se es) en función del nivel de competencia que es esperable poseer en un momento concreto de la formación y el 
desarrollo de las personas. Nicoli (2000) ha insistido en ese sentido evolutivo y progresivo de las competencias diferenciando 5 niveles de dominio: la mera ejecución de algo; la ejecución con un relativo nivel de autonomía; la ejecución de tareas en un contexto de complejidad, la complejidad unida a la dirección de personas; la creación de nuevos modelos de actuación. Puesto que hablamos de estudiantes, este sentido relativo y circunstancial de las competencias resulta fundamental: las competencias de un profesional de la arquitectura no son las que cabe esperar de un estudiante de arquitectura. Por eso difieren los niveles de dominio exigibles en el grado, el máster o el doctorado. Las competencias son ámbitos de dominio que se organizan como un continuum progresivo e interminable (por eso hablamos del lifelong learning). Trabajar por competencias en la universidad exige ser capaces de establecer un "grado de dominio" de la competencia para ese momento de la formación (Kinchin, Cabot \&Hay, 2006), partiendo del nivel ya alcanzado y teniendo en mente los niveles de dominio que seguirán a los que los estudiantes deberán alcanzar con nosotros

\section{Evaluar competencias en la universidad}

Visto lo que tienen de propio y característico en la formación universitaria tanto la evaluación como las competencias, podemos pasar ahora a señalar, con la misma lógica empleada en los apartados anteriores, las condiciones de la evaluación de competencias.

\subsection{Si las competencias suponen la puesta en acción de un conocimiento, está claro que la vía de acceso a la competencia ha de hacerse a través del componente ejecutivo del aprendizaje}

Lo que el aprendiz hace en una situación determinada en función de lo que sabe y siente. Ala valoración del dominio ejecutivo se puede entrar por 4 vías: (a) el ajuste y precisión (si el estudiante ha captado la lógica de una acción y es capaz de repetirla sin alteraciones. Es una evaluación basada en la fidelidad al procedimiento): el evaluador observa la realización y constata si se ajusta a lo esperado; (b) la eficacia del desempeño (si el estudiante realiza su tarea de forma eficaz obteniendo resultados positivos y/o resolviendo el problema): se valora la eficacia y la utilidad del desempeño no que se haya seguido el protocolo previsto; (c) la contextualización (si el estudiante toma en consideración los elementos variantes del contexto en el que debe actuar y acomoda a ellos su respuesta); (d) la originalidad (si el estudiante desarrolla modos singulares de enfocar los problemas y diseñar su actuación). Sin duda, la vida profesional les va a exigir actuaciones de los 4 tipos en función del tipo de actividad que en cada caso deban realizar. No está de más, por tanto, que también durante la formación se busquen momentos en los que cada competencia deba abordarse desde estos planteamientos complementarios.

4.2 Decíamos al hablar de la evaluación que esta requiere evidencias y, por tanto, dispositivos que nos permitan documentar elementos relevantes de la actuación de los estudiantes en cada una de las competencias trabajadas

En ese sentido, la presencia de dichos dispositivos no es neutral y, como se deriva del principio de indeterminación de Heisenberg, condicionan los resultados de la evaluación. Le Boterf (2005) es lapidario al respecto: "Es preciso tomar en consideración que el evaluador evalúa no las competencias sino lo que el dispositivo de evaluación designa por competencias" (p. 62). Al final, el dispositivo de evaluación que se utilice no es sino la síntesis final de todo el proceso de configuración de la evaluación: que se entiende por competencia, qué 
aspectos y/o conductas se van a tomar como relevantes, en relación a qué referentes se van a evaluar, en qué situación se va aponer al estudiante para que demuestre su dominio, etc. Por eso es bien diferente una evaluación basada en la observación y operada a través de un check list, a una observación mediante pruebas estándar, a la actuación libre frente a una situación real (un paciente, por ejemplo), a un ejercicio libre en relación con una cuestión abierta.

4.3 Las competencias pueden concebirse como actuaciones más simples (la ejecución correcta de algo) o más complejas (un proceso que incluye fase de planeación, de ejecución y de valoración de lo que se hace)

Aunque el contenido de la competencia dependerá de la naturaleza de los aprendizajes que se estén abordando, lo natural es que la formación universitaria tienda a trabajar competencias complejas que vayan más allá de la mera ejecución de actividades prediseñadas. Competencias en las que el estudiante deba poner en juego sus conocimientos para construir una respuesta adaptada a las circunstancias del contexto en el que haya de desarrollar la acción. Y si los conocimientos y las habilidades precisas para llevar a cabo la actividad demandada provienen de diferentes campos disciplinares, esa sería la situación de evaluación más rica y adecuada a los propósitos formativos de la universidad.

\subsection{Algo importante a tomar en consideración en la evaluación de competencias es que no existen en abstracto (o existen solo como constructo conceptual)}

Las competencias son realidades invisibles que se manifiestan a través de respuestas competentes. Las competencias suponen una forma de actuar. De nadie podemos decir que es competente sin verlo actuar puesto que ser competente supone llevar a cabo actuaciones competentes. Es decir, la competencia existe en el sujeto pero, necesariamente, ha de mostrarse fuera de él. Y es en esa epifanía, en esa salida al exterior cuando la competencia deja de ser una cualidad personal (el sujeto competente) para convertirse en una realidad social (la conducta competente) que ya no depende solo del sujeto que la posee sino de las condiciones de la situación en que la competencia ha de manifestarse. Jonaert (2006) ha insistido en la importancia de la noción de situación para poder entender y evaluar las competencias, tanto por lo que la situación exige al sujeto (él habla de la inteligencia de la situación) como por el efecto que la propia situación puede tener sobre la performance a realizar por el estudiante. Para este autor canadiense, el "tratamiento eficaz de las situaciones" (p.4) es el principal componente de una competencia.

Esa circunstancia mixta (sujeto + situación) constituye una condición fundamental para la evaluación de competencias. Pensemos en alguien que tiene que demostrar su competencia comunicativa o su habilidad artística o su capacidad de liderazgo, pero debe hacerlo en un contexto material u organizativo deficiente. Con seguridad, su performance (lo que nosotros vamos a ver $y$, por tanto, evaluar) va a ser mediocre pero no por que lo sea la competencia sino porque su manifestación se ha visto contaminada por las condiciones de la situación en que debía visibilizarse. Y eso requiere que toda evaluación de competencias debe propiciar condiciones adecuadas para que la competencia pueda manifestarse sin limitaciones que la alteren. 


\subsection{Un aspecto relevante de la formación universitaria es que se trata, justamente, de un periodo de formación}

Nuestros estudiantes no son profesionales, se preparan para serlo. No tienen que (no podemos exigirles) actuar como profesionales, sino como sujetos que quieren serlo y se esfuerzan, aprenden, para serlo. Es decir, lo importante no es tanto que hagan las cosas bien cuanto que se den cuenta de cómo las hacen y puedan corregirlas si no están bien. Este componente reflexivo de la formación basada en competencias resulta fundamental y debería constituir una parte fundamental de la evaluación. Pretendemos valorar no solo la dimensión ejecutiva de la competencia (cómo lo ha hecho), sino también la dimensión narrativa del proceso (cómo lo ha diseñado y ejecutado, el porqué de las decisiones que ha ido tomando). La respuesta competente, dice Le Boterf (2005, p. 68), "debe poder contarse, ser objeto de una narrativa. Es la narrativa del proceso de construcción de una respuesta competente, de una ética profesional competente".

\section{La experiencia de la PUCMM en la implementación y evaluación de competencias}

\subsection{Contexto del caso}

El Procedimiento CG-CEA: de las competencias generales a las competencias específicas de asignatura (Lodeiro, 2017) se centra en la parte operativa, combinando el pragmatismo derivado de la necesidad de diseñar los planes de estudio por competencias con una fuerte intencionalidad formativa. Tanto para las competencias generales como para las específicas de titulación, se contempla el establecimiento de unos niveles de dominio o resultados de aprendizaje que constituyen una secuencia interna de complejidad en el desarrollo de la competencia.

Esos niveles de dominio de las diferentes competencias se utilizan como la unidad funcional del plan de estudios y, tras haber analizado las relaciones temporales entre ellos, se distribuyen entre las diferentes asignaturas, dando lugar al mapa de competencias. De este modo, también se está distribuyendo la responsabilidad con respecto al perfil del egresado, pues cada asignatura se ocupa de trabajar y evaluar, al menos, un nivel de dominio de una competencia general y un nivel de dominio de una competencia específica. Ya indicados los niveles de dominio a trabajar en cada materia, se concretan en su programa las competencias específicas de asignatura que reflejan comportamientos observables y medibles en los que se concretan los niveles de dominio adjudicados a la misma.

Este procedimiento se ha diseñado, validado y mejorado a través de tres años (febrero de 2014 - febrero de 2017) de experiencia de una asesora curricular en la Pontificia Universidad Católica Madre y Maestra (PUCMM) y, por tanto, toma como referencia inicial el marco establecido por esta universidad de República Dominicana. En este caso, el proceso del diseño original de una carrera por competencias se divide en dos fases: en la primera, una comisión redactora se encarga de elaborar un núcleo que contiene los componentes generales del proyecto educativo (perfil del egresado, malla curricular, mapa de competencias, orientaciones metodológicas, recursos necesarios para la implementación, etc.) y, en la segunda, se implican más profesores para diseñar de forma escalonada los programas de las asignaturas. En los tres años de implementación del 
Procedimiento CG-CEA en la PUCMM se trabajó con 14 comisiones redactoras que implicaron a un total de 88 docentes para el diseño de 17 núcleos de planes de estudio. Hasta el momento los esfuerzos se concentraron en la urgencia del diseño sin poder realizar un seguimiento institucional sistemático de la implementación que, aunque también importante y urgente, no era tan inminente.

\subsection{Recopilación de la información y muestra}

Por lo expuesto anteriormente, y con la intención de hacer una primera exploración sobre cuáles son las impresiones con respecto al desarrollo de las competencias y su evaluación que emanan de la experiencia de los docentes, se realizaron diez entrevistas en profundidad (de aproximadamente una hora de duración) a una muestra de profesores que imparten o han impartido alguna asignatura por competencias en la PUCMM.

Los diez profesores entrevistados trabajan en carreras como estomatología, terapia física, interiorismo, arquitectura, comunicación corporativa, comunicación audiovisual, psicología, ingeniería civil, ingeniería mecánica e ingeniería eléctrica. Siete de ellos pertenecieron a la comisión redactora de las carreras en las que imparten clase y nueve pasaron por la experiencia de diseñar algún programa de asignatura por competencias.

Uno de los entrevistados tiene cinco años de experiencia dando clase en la institución, pero los demás superan la década e, incluso, las dos décadas en el caso de seis de ellos. Sin embargo, su experiencia en la implementación de asignaturas por competencias es todavía breve, oscilando entre los cuatro meses y los dos años. Dado que la Universidad ha comenzado a incursionar recientemente en procesos de acreditación internacional de sus titulaciones, dos de los entrevistados están implicados en una carrera que se encuentra en proceso de acreditación.

\subsection{Resultados}

Aunque las entrevistas realizadas contemplan varias dimensiones, en esta ocasión nos centraremos fundamentalmente en resumir las impresiones que los participantes compartieron con nosotros sobre el modo en que entienden la formación por competencias y las diferencias que, a través de su experiencia, perciben entre esta forma de trabajar y el sistema anterior. Todo ello centrado principalmente en la evaluación: cómo se diferencia de las que realizaban anteriormente, las dificultades que entraña, las oportunidades que ofrece y los retos que plantea. A lo largo del análisis se incorporan algunos fragmentos de las transcripciones de las entrevistas.

\subsubsection{Influencia de la experiencia de diseño por competencias en la experiencia de implementación}

Los integrantes de comisiones redactoras indican que para ellos es más sencillo que para el resto del profesorado diseñar programas de asignaturas y llevar a cabo la implementación de los mismos. Explican que haber ideado las competencias de la carrera con sus correspondientes niveles de dominio y haber participado en las diferentes actividades para llegar al mapa de competencias, les ayudó a entender mejor el modelo y sus implicaciones didácticas, así como el sentido global del proyecto educativo. Eso los lleva a tener una actitud más abierta y les permite avanzar más rápido. Sin embargo, reconocen que, al igual que los demás, necesitan más formación sobre estrategias didácticas y de evaluación acordes con el trabajo por competencias.

Todo esto es más sencillo para las personas que participaron en la Comisión. Avanzan a mejor ritmo y con mejor actitud porque entienden mejor en qué consiste trabajar por 
competencias. Pero también necesitan acompañamiento en la implementación. (Entrevista número 4)

\subsubsection{Necesidades y condiciones}

Ocho de los entrevistados manifiestan que, como parte de la formación, el conjunto del profesorado necesita dos tipos de acompañamiento: (a) acompañamiento sincrónico mientras diseñan los programas, preparan los recursos e instrumentos de evaluación, implementan las asignaturas y evalúan a los estudiantes; (b) acompañamiento diacrónico orientado al análisis de lo sucedido y la incorporación de mejoras.

Proponen que las personas encargadas de realizar esos acompañamientos sean docentes de la misma carrera, de tal modo que entiendan la idiosincrasia de la profesión y les ayuden a entender cómo se conectan las competencias generales y las específicas. Necesitan retroalimentación también para saber si están haciendo las cosas bien en función de lo consignado en el modelo educativo de la Institución.

Los profesores necesitan acompañamiento sincrónico (mientras diseñan, implementan las
asignaturas y evalúan a los estudiantes) y diacrónico con el propósito de analizar lo sucedido
e introducir mejoras. Preferiblemente un acompañamiento por parte de personas del mismo
departamento que entiendan la idiosincrasia de la carrera y ayuden a los profesores a entender
cómo se conectan las competencias genéricas y las específicas. Necesitan retroalimentación
también pasa saber si están haciendo las cosas bien. (Entrevista número 4)

Esto nos indica que sienten cierta inseguridad, acentuada en el caso de los profesores que no formaron parte de una comisión redactora. No obstante, de esa inseguridad están surgiendo interesantes iniciativas en los departamentos encaminadas a desarrollar procesos reflexivos para compartir experiencias entre pares con el propósito de apoyarse y buscar soluciones a los problemas detectados.

En el Departamento se creó una coordinación de evaluación de la calidad que apoya al profesorado en la planificación e implementación. (...) la coordinación de evaluación de la calidad ofrece retroalimentaciones a los docentes basadas en los resultados obtenidos. (Entrevista número 1)

(...) nos vamos a reunir como departamento para compartir experiencias e intentar reducir la cantidad de actividades de evaluación. (Entrevista número 4)

\subsubsection{El compromiso y la responsabilidad como elementos esenciales}

Casi la totalidad de los entrevistados llamaron la atención sobre el hecho de que, cuando una IES toma la decisión de cambiar su modelo educativo, debe hacerlo consciente de las implicaciones logísticas y económicas de la decisión. Es decir, asumir el compromiso y la responsabilidad institucional en lo que se refiere a la aplicación, el control de calidad y la evaluación del mismo. En este sentido, los profesores ponen de manifiesto que, para trabajar y evaluar competencias de forma genuina, la ratio profesor-alumno no debe ser superior a 20 estudiantes por grupo y deben adquirirse todos los recursos que en los planes de estudio se han consignado como imprescindibles para viabilizar la implementación.

\footnotetext{
Se necesita compromiso institucional con el modelo y su desarrollo. Está faltando apoyo y seguimiento. No se está atando esto al proceso general, analizando durante la implementación cuán real es el plan de estudios y su viabilidad. Se trata de un compromiso económico también. (Entrevista número 1)

Me preocupa que se pueda conseguir en asignaturas que requieren de recursos que la Universidad no ha proporcionado todavía. (Entrevista número 8)
}

Esa idea del compromiso y la responsabilidad es recurrente en todas las entrevistas realizadas llevándola más allá de los órganos de decisión de la Universidad. Entienden que 
se trata de un modelo con muchas virtudes, pero, en ausencia de un mecanismo de sistematización de evidencias y control de la calidad, todas ellas están supeditadas al nivel de compromiso y responsabilidad asumido tanto por los estudiantes como por los profesores.

Por una parte, consideran que todavía son muchos los docentes que se están resistiendo al cambio y continúan haciendo las cosas como las hacían antes, mientras otros son muy permisivos con los estudiantes y no mantienen cierto nivel de exigencia buscando su aprecio. Por otra parte, explican que el nuevo planteamiento de formación es una buena oportunidad de aprendizaje con excelentes resultados en aquellos estudiantes que se implican responsablemente en su proceso. Pero, de igual manera, a aquellos que no asumen ese compromiso con su aprendizaje, ahora les resulta más fácil obtener unas buenas calificaciones sin haber demostrado realmente el nivel de desempeño requerido. Esto pone en entredicho el cumplimiento de la función acreditadora de las evaluaciones que se están realizando.

\subsubsection{Cambios en el sistema de evaluación}

Con respecto al sistema de evaluación, señalan tres cambios que creen pueden arrojar luz para comprender el origen del fenómeno que acabamos de mencionar:

- Tipo de actividades. Pasaron de una evaluación en la que la calificación dependía casi exclusivamente de exámenes parciales y finales (basados en la reproducción de información y la resolución de algún ejercicio práctico) a otra en la que priman otras actividades como, por ejemplo, la realización de un proyecto o un portafolio.

Mi tabla de evaluación antes tenía 5 columnas, ahora tiene 10 (y porque agrupo cosas). Ahora son menos exámenes, esos exámenes representan un menor porcentaje de la evaluación (entre un 30 y un 40 \%) y, además, a veces son diferentes. Por ejemplo, ahora estoy haciendo algún examen grupal de desarrollo en el que los grupos de trabajo tienen que discutir y ponerse de acuerdo para escribir las respuestas. (Entrevista número 9)

- Lugar en el que se realizan las actividades. Los exámenes normalmente se hacen en el aula bajo la supervisión del profesor, de tal modo que éste puede tener casi la certeza de que, al menos, el que escribe o habla es el propio estudiante que está evaluando. También algunas simulaciones permiten este control. Sin embargo, en el caso de los proyectos, portafolios, investigaciones y demás actividades; la mayor inversión de horas es de aquellas contabilizadas como de trabajo autónomo de los estudiantes y se escapan de la "vigilancia” del docente. Informan de que ya no basta con solicitar trabajos lo suficientemente originales como para que los estudiantes no puedan copiados, ahora ya existen anuncios en internet en los que se ofrecen servicios para realizar un trabajo concreto de una asignatura específica según las consignas del momento.

De lo que más se habla es de que cambió la evaluación: en lugar de un examen ahora tengo un proyecto o un portafolio. El problema es que los muchachos copian los trabajos o pagan a alguien para que se los haga, hay un gran problema en los estudiantes que tiene que ver con la honestidad. Es una cuestión cultural también, incluso hay anuncios por internet en los que alguien publicita que se realizan portafolios para asignaturas concretas de la PUCMM. La evaluación de esta forma se escapa un poco al control del profesor, al menos en los exámenes tienes a los estudiantes en el aula haciendo las cosas delante de ti, es más fácil con los exámenes saber que a quien estás evaluando es a tu estudiante. Todo esto además sobrecarga al profesor al tener que revisar más proyectos y trabajos al mismo tiempo que tiene que vigilar el asunto del plagio. En sintesis, se trata de una forma diferente de hacer las cosas. Puede funcionar, 
pero solamente si tanto el profesor como el estudiante ponen de su parte, asumen sus respectivas responsabilidades y basan su comportamiento en la honestidad. (Entrevista número 8)

- Actividades grupales. Fruto de inclusión de competencias generales como el trabajo en equipo y de la sobrecarga que supone para los docentes evaluar a través de proyectos de mayor extensión con la misma cantidad de estudiantes, se incluyen entre las actividades de evaluación muchas tareas grupales. Esto implica que algunos estudiantes puedan aprovecharse del trabajo de sus compañeros e induce a los profesores a pensar que es necesario reducir la cantidad de actividades grupales y recibir formación sobre estrategias de evaluación que les permitan discriminar mejor el desempeño individual de cada estudiante sin sobrecargarse todavía más.

Se necesita formación para el profesorado con el propósito de que comprendan mejor el modelo, sus implicaciones didácticas y estrategias de evaluación acordes con el modelo que pudieran no sobrecargar tanto a los profesores. (Entrevista número 1)

\subsubsection{Función acreditadora de la evaluación}

Cuando preguntamos directamente a los entrevistados si creen que las evaluaciones que se están llevando a cabo en las carreras por competencias de su Universidad cumplirán la función acreditadora, solamente obtenemos una respuesta afirmativa. Los demás dudan y consideran que esa función se cumplirá solo parcialmente. Esa respuesta neutra la completan indicando que no saben hasta qué punto el conjunto del profesorado está diseñando programas de asignaturas fieles a la esencia del núcleo o hasta qué punto lo que hacen con los estudiantes se corresponde con lo planificado en los programas de asignaturas fieles al núcleo. También muestran preocupación por el cumplimiento de las expectativas en aquellas asignaturas para las que todavía no se han adquirido los recursos técnicos imprescindibles.

\section{Al no haber un mecanismo de control de la calidad en la Universidad la distancia entre los papeles y lo implementado es tan grande como los profesores quieran. (Entrevista número 6) \\ (...) los profesores están sobrecargados porque la misma cantidad de créditos implica ahora mucho más trabajo de planificación y evaluación. (Entrevista número 7)}

Apuntan que las pasantías bien planificadas y las tesis o trabajos fin de grado (si los hacen los propios estudiantes) podrían convertirse en un buen momento para comprobar hasta qué punto se ha alcanzado el perfil profesional planeado. Además, la existencia de un apoyo más directo a los profesores y la creación de un mecanismo de control de la calidad podrían contribuir a lograr la meta. Precisamente, los entrevistados implicados en una carrera inmersa en un proceso de acreditación internacional declaran que, en su departamento, no se estaría guardando un registro sistemático de evidencias si no fuera por esa intención acreditadora.

Las pasantías se pueden convertir en el medio para comprobar el nivel de adquisición de las competencias si se preparan los programas de las mismas con rúbricas con indicadores de logro de cada nivel de dominio. (Entrevista número 7)

\subsubsection{Función formativa de la evaluación}

$\mathrm{Al}$ focalizar la entrevista en los cambios que los informantes consideran que el nuevo modelo provocó en la evaluación, dan respuestas que nos llevan a pensar que, desde su punto de vista, la función formativa está cobrando protagonismo. Creen que ahora se trata de evaluar las producciones (o resultados finales) que generan los estudiantes, pero, sobre todo, de hacer un seguimiento de su evolución en el proceso, ofreciéndoles retroalimentaciones que les permitan comprender las implicaciones de lo que hacen, 
aprovechar el valor pedagógico del error y ser conscientes de su progresión en el aprendizaje.

Los estudiantes pueden localizar con facilidad sus puntos fuertes y débiles dado que están muy conscientes de su proceso y de su medición. Se puede medir el proceso y eso es un gran aporte del modelo porque existe una retroalimentación constante. (Entrevista número 10)

Antes la evaluación se hacía a través de exámenes finales focalizados en el temario de la asignatura ahora la evaluación es más formativa y sistemática, implica hacer retroalimentaciones a lo largo del proceso. (Entrevista número 4)

Por ejemplo, uno de los entrevistados nos cuenta que, en el tiempo que lleva dando clase de asignaturas por competencias, descendió la cantidad de estudiantes que solicitan revisiones de sus calificaciones, hasta el punto de no haber recibido ninguna solicitud en asignaturas por competencias. Ese mismo profesor comenta que introduce un examen oral al final de la asignatura en el que pregunta a los estudiantes qué calificación consideran que merecen. Las respuestas que obtiene siempre coinciden con la calificación que él mismo considera que el estudiante merece. Esto es, explica, porque está todo tan transparentado y claro, que los estudiantes saben exactamente lo que se está requiriendo de ellos en cada momento.

\begin{abstract}
Bajó espectacularmente la cantidad de revisiones que solicitan los estudiantes. De hecho, en las asignaturas por competencias nunca he tenido una revisión de notas. (...) To hago un examen oral a los estudiantes y una de las cosas que les pido en ese examen es que analicen su proceso y su evolución y me digan qué calificación consideran que se merecen y siempre coincide su nota con la mía. (Entrevista número 10)
\end{abstract}

Esta transparencia y claridad no es percibida por todos los entrevistados, algunos nos explican que todavía la evaluación es un misterio para ellos, que los indicadores incluidos en las rúbricas de evaluación son demasiado generales y resulta complicado concretarlos en los contenidos o que sienten que no está todo bien atado.

Queda la inquietud sobre la relación entre los diferentes tipos de competencias del programa (las genéricas, las específicas de titulación y las específicas de la asignatura) y la parte de la evaluación. Porque hay muchas cosas que pueden pasar, pero no las sabemos, en la implementación del programa deberíamos trabajar con ellas, pero no las conocemos. El tema de la evaluación es un punto misterioso para mí. (Entrevista número 8)

\title{
5.3.7. El papel de las competencias generales
}

A pesar que todavía no tienen muy claro cómo trabajar y evaluar las competencias generales (sobre todo las que tienen más carga actitudinal) o cómo combinarlas con las específicas de titulación, no creen que la solución pase por eliminarlas del perfil. Por el contrario, consideran que las competencias genéricas son un valor agregado en la formación universitaria, sobre todo en un contexto en el que la corrupción está tan presente y hay una gran cantidad de profesionales a los que no les importan los demás. No pueden cambiar la personalidad de los estudiantes, pero, insisten, sí pueden tratar de ofrecerles un proceso mejor, por eso insisten en que necesitan ser formados para poder afrontar el reto.

De manera tradicional estábamos acostumbrados al aprendizaje de la disciplina y ahora con las competencias genéricas le agregamos el desarrollo integral de la persona y eso es un aporte importante. Probablemente antes también se trabajaba alguna competencia genérica pero no de manera formal; ¿̇Por qué tenemos en el País tantos profesionales a los que no les importan los demás?; ¿̇e qué nos vale tener personas que son muy buenas en su disciplina si no son respetuosas o no muestran un comportamiento ético?; No podemos cambiar la personalidad de los estudiantes, pero sí podemos generar un proceso mejor. (Entrevista número 4) 
Ven una correlación directa entre las competencias generales y las específicas pues, en realidad, las segundas dependen de las primeras, las necesitan para desarrollarse plenamente. Una de las entrevistadas nos aporta un ejemplo en este sentido:

De poco valdría a un egresado saber hacer el diagnóstico en psicología (competencia específica) si no puede reflexionar sobre las informaciones obtenidas y dar recomendaciones. Para hacer todo eso necesitan haber desarrollado competencias genéricas como el pensamiento reflexivo, el pensamiento crítico o el pensamiento sistémico. (Entrevista número 7)

\subsubsection{Efectos del cambio en las calificaciones de los estudiantes}

Ninguno de los consultados percibió que las calificaciones de los estudiantes hayan bajado y ocho aseguran que han subido. Entre las causas a las que atribuyen el incremento se encuentran las siguientes:

a) al solicitar más trabajos grupales unos estudiantes tiran de otros,

b) al realizar una evaluación más formativa los estudiantes tienen más oportunidades para mejorar y entienden mejor el proceso,

c) la variedad de estrategias metodológicas aumenta las posibilidades de llegar a más estudiantes,

d) el componente práctico es más motivador para los estudiantes porque perciben con facilidad la utilidad de lo que están aprendiendo

e) la puntuación está más dispersa en diferentes actividades a lo largo de la asignatura $\mathrm{y}$

f) al realizar una evaluación del proceso los estudiantes no sienten tanta presión como cuando todo dependía del momento puntual en el que se realizaban unos exámenes.

Las calificaciones mejoraron y puede deberse a la cantidad de puntos dispersos. También aumenta el nivel de compromiso de los estudiantes. (Entrevista número 2)

Mejoraron las calificaciones por las estrategias que se están utilizando. Ahora el proceso es más llevadero, es más fácil obtener calificación y se logran aprendizajes de mejor calidad. (Entrevista número 4)

Como se hacen muchos trabajos colaborativos al estudiante se le hace mucho más fácil pasar la asignatura sin tener las competencias. El profesor no tiene tiempo para verificar el nivel de desempeño de cada estudiante. Se le está dando mucho peso al trabajo en grupo y se pierde un poco la evaluación individual puesto que las pruebas individuales se recuden mucho. (...). Las calificaciones son ligeramente mejores y creo que se debe a que hay más trabajos colaborativos (con las implicaciones que explicaba antes). (Entrevista número 7)

To creo que mejoran y que, en parte, se debe a que no depende todo de unos exámenes finales de los que depende todo y en los que, por el simple hecho de ponerse nerviosos, puede llevar a que obtengan peores calificaciones. Además, al combinar diferentes estrategias y métodos de evaluación hay más posibilidades de dar con el estilo de aprendizaje del estudiante. (Entrevista número 9).

Sí, obtienen mejores calificaciones porque no se evalúa solamente el resultado de un examen final en un examen que completan con presión. Como se evalúa el proceso eso implica menos presión y permite ofrecer retroalimentaciones que proporcionan a los estudiantes oportunidades de mejora. (Entrevista número 1)

\subsection{Conclusión general}

Tras haber hecho un breve recorrido por las impresiones que los docentes compartieron con nosotros podríamos concluir que nos encontramos ante un caso esperanzador. No 
consideran que estén trabajando con el modelo perfecto, tampoco que lo estén haciendo todo lo mejor posible, pero parecen mantener una actitud reflexiva y crítica que les permite identificar los problemas, analizarlos en busca de posibles causas, formular los retos que tienen y proponer posibles cursos de acción para aproximarse, poco a poco, al cumplimiento de sus expectativas.

\section{Las Competencias como síntesis final de la formación recibida: las ECOEs (Evaluación Clínica Objetiva Estructurada) de las Facultades de Medicina}

Nos interesa mucho incorporar a este apartado final el caso de las ECOEs, introducido en numerosas Facultades de Medicina porque representa una perspectiva netamente institucional y metadisciplinar de las competencias y su evaluación. Frente a la tendencia general a vincular las competencias a las disciplinas individuales, las ECOEs buscan situaciones más globales y, por tanto, analizan performances que requieren el dominio de situaciones complejas y la integración de aprendizajes provenientes de diferentes disciplinas. Por otro lado, está llamada a fortalecer alineamiento entre la evaluación de competencias y la acreditación de la formación recibida, aunque hemos de reconocer que aún estamos lejos de alcanzar ese objetivo.

Basada en la pirámide de Miller (1990) que estructura el conocimiento práctico en cuatro niveles sucesivos: saber, saber decir cómo se hace, saber hacerlo en situaciones simuladas, saber hacerlo en la vida real, se entiende que las ECOEs servirían para cumplimentar el tercero de dichos niveles. Los estudiantes han de enfrentarse a un conjunto de situaciones clínicas sencillas que deben resolver. Las pruebas se realizan en diferentes estaciones (en España suelen ser 20) en cada una de las cuales se le plantea diferentes situaciones médicas con pacientes reales (normalmente actores) o a través de documentación clínica (una imagen, un informe médico, un fallecimiento para hacer el parte de defunción, etc.).

Son 7 las competencias que se incorporan a las pruebas ECOE: habilidades clínicas básicas (anamnesis, por ejemplo); comunicación médico-paciente; habilidades técnicas; manejo del problema; atención a la familia; actividades preventivas; atención a la comunidad; docencia; investigación; aspectos organizativos. No todos estos aspectos se analizan en cada una de las estaciones y los que se analizan y valoran pueden poseer distinto peso según la naturaleza del asunto que se deba afrontar en cada una de las estaciones. Si se trata de hacer una sutura el peso mayor lo llevarán las habilidades técnicas, pero en el caso de un dolor abdominal los pesos mayores pueden estar en la anamnesis y la comunicación con el paciente. En cualquier caso, qué aspectos se priorizarán en cada situación lo decide el equipo gestor de las pruebas y puede variar de un estudiante a otro.

La gran virtud de las ECOEs es el compromiso institucional que suponen y el hecho de que exigen a los estudiantes integrar aprendizajes teóricos y prácticos de materias diversas para aplicarlos a la resolución de un problema específico. La evaluación de cada una de las estaciones se hace a través de un protocolo (una rúbrica) previamente establecido cuyos ítems los estudiantes desconocen y pueden variar de unos a otros. Por lo general, el objetivo central de la evaluación no es constatar si el estudiante ha dado la respuesta correcta (si ha acertado el diagnóstico, si ha diseñado el tratamiento adecuado, etc.). Dan más valor a la forma en que ha afrontado la situación, a si ha hecho las preguntas 
correctas al paciente, si se ha comunicado con él de forma aceptable, si ha seguido la lógica propia de la profesión médica. Esto es, se trata de integrar de forma consistente las competencias más específicas con las transversales y generales de la clínica.

La parte débil de las ECOEs es su gestión administrativa. Todavía, al menos en lo que se refiere a las universidades españolas en general, este procedimiento de evaluación de competencias no ha encontrado su sitio en el diseño de la formación médica. Se le ha convertido en una asignatura más o se le ha integrado en otra preexistente con lo cual, acaba desvinculándose del conjunto del currículo. No puede plantearse como una evaluación estándar porque eso les obligaría a hacer dos convocatorias (algo que haría insostenible el modelo: los estudiantes a evaluar pueden rondar los 4.00 cada año en cada Facultad); se valoran con un simple apto-no apto (en los 6 años de vigencia del modelo nadie ha sido valorado hasta la fecha como no apto) porque ponerle nota significaría impactaría en su calificación total y afectaría a su examen MIR. Desde el punto de vista del reconocimiento de quienes actúan en él se le ha prestado poco valor: su valor docente es escaso y se toma poco en cuenta la gran dedicación que exige. Con todo, supone un gran mérito para las Facultades de Medicina que lideradas por la Conferencia Nacional de Decanos de Medicina ha logrado ponerla en marcha en todas las Universidades y trabaja ahora para homogeneizarla (van a necesitar acreditarse ante una comisión reguladora) en todas ellas y convertirla en una fuente de información sobre el desarrollo de la formación médica en España. Mérito este a destacar pensando que ese mismo proceso podría haberse producido en otras Facultades, pero no se ha logrado.

\section{Referencias}

Barnett, R. (2001). Los límites de la competencia. El conocimiento, la educación superior y la sociedad. Barcelona: Gedisa.

Boud, D. y Falchikov, N. (2006). Aligning assessment with long-term learning. Assessment \& Evaluation in Higher Education, 31(4), 399-413. https://doi.org/10.1080/02602930600679050

Brown, S. y Glasner, A. (2003). Evaluar en la Universidad. Problemas y nuevos enfoques. Madrid: Narcea.

Cano, E. (2008). La evaluación por competencias en la Educación Superior. Profesorado. Revista Curriculum y Formación del Profesorado, 12(3), 1-16.

Conan, J. (1999). A Handbook of Techniques for Formative Evaluation: mapping the student's learning experience. Londres: Kogan Page.

Fletcher, S. (1997). Analysing Competence: tools and techniques for analyzing jobs, roles and functions. Londres: Kogan Page.

Hager, P., Gonczi, A. y Athanasou, J. (1994). General Issues about Assessment of Competence. Assessment $\Xi^{2}$ Evaluation in Higher Education, 19(1), 3-16.

Houston, W. R. (1985). Competency-based Teacher Education. En T. Husen y T. Neville Postlethwaite (Eds.), International Encyclopedia of Education (pp. 898-906). Oxford: Pergamon.

Jonnaert, Ph., Barrette, J., Masciotra, D. y Yaya, M. (2006). Revisión de la competencia como organizadora de los programas de formación: hacia un desempeño competente. Montreal: Univ. de Quebec, Observatorio de Reformas Educativas. 
Kinchin, I. M., Cabot, L. B. y Hay, D. B. (2006, diciembre). The variable structure of expertise: Implications of differences between practitioners and researchers for university teaching. Comunicación presentada en la Society for Research into Higher Education (SRHE) annual conference, Universidad de Brighton, Reino Unidp.

Le Boterf, G. (2005). Construir as Competências Individuais e Colectivas. Oporto: ASA Editores.

Lodeiro, L. (2017). Procedimiento CG-CEA: de las competencias generales a las competencias específicas de asignatura. Manual del asesor curricular. Documento inédito.

Miller, G. E. (1990). The assessment of clinical skill/competence/performance. Academic Medicine, 65(9), 563-567.

Nicoli, D. (2000). Natura della competenza e suoi possibili utilizzi. Una proposta di modello di competenza. En S. Cortelazzi y I. Pais (Coord.), Il posto della competenza (pp. 47-89). Milán: Franco Angeli.

Qualifications and Curriculum Authority (QCA). (2007). E-Assessment. Guide to Effective Practice. Recuperado de www.e-assessment.com/wp-content/uploads/2014/08/e-assessment__guide_to_effective_practice_full_version.pdf

Rodríguez-Gómez, G. e Ibarra, M. S. (2011). E-Evaluación orientada al e-Aprendizaje estratégico. Madrid: Narcea.

Sanz de Acevedo, M. L. (2016). Competencias cognitivas en educación superior. Madrid: Narcea.

Serdio Romero, E. (2002). ECOE. Evaluación clínica objetiva estructurada. Medicina de Familia, 2, 127-132.

Villardón-Gallego, L. (2015). Competencias genéricas en Educación Superior. Metodologías específicas para su desarrollo. Madrid: Narcea

Yániz, C. (2008). Las competencias en el currículo universitario: implicaciones para diseñar el aprendizaje y la formación del profesorado. REDU, Revista de Docencia Universitaria, 6(1), 114. https://doi.org/10.4995/redu.2008.6281

Zabalza, M. A. (2003). Competencias docentes del profesorado universitario. Madrid: Narcea.

Zabalza, M. A. y Zabalza Cerdeiriña, M. A. (2018). Planificación de la docencia en la universidad. Elaboración de las guías docentes de las materias. Madrid: Narcea.

\section{Breve CV de los autores}

\section{Miguel A. Zabalza Beraza}

Doctor en Psicología por la Universidad Complutense de Madrid. Catedrático de Didáctica y Organización Escolar de la Universidad de Santiago de Compostela y profesor visitante de universidades de Europa e Iberoamérica. Director del grupo de investigación GIE (GI-1444), especializado en temas de docencia universitaria. Presidente de la Asociación Iberoamericana de Docenci a Universitaria (AIDU). Ha publicado, individualmente o en colaboración, más de 100 libros de diversas problemáticas educativas. ORCID ID: 0000-0002-9490-5438. Email: miguel.zabalza@usc.es

\section{Laura Lodeiro Enjo}

Licenciada en Psicopedagogía y Maestra de Educación Infantil. Ha sido premio de Fin de Carrera de la Universidad de Santiago de Compostela y de la Comunidad Autónoma de 
Galicia en sus dos titulaciones universitarias y ha obtenido la máxima calificación (Sobresaliente Cum Laude) en su tesis doctoral sobre los equipos docentes en Educación Superior. Es coeditora del libro Equipos Docentes y nuevas Identidades Académicas publicado en 2010 y, como integrante del Grupo de Investigación Grupo Interuniversitario de Estudios (GIE) dirigido por el Prof. Miguel Ángel Zabalza, ha participado en diferentes proyectos de investigación sobre docencia universitaria. Desde enero de 2014 desarrolló su actividad profesional en la Pontificia Universidad Católica Madre y Maestra (PUCMM) como Encargada Corporativa de la Unidad de Diseño del Centro de Desarrollo Curricular (CDC). Ha diseñado el Procedimiento CG-CEA (de las competencias generales a las competencias específicas de asignatura) y, aplicándolo, ha asesorado a las comisiones redactoras de 21 planes de estudio por competencias en su Universidad. Actualmente es la Directora del Centro de Desarrollo Curricular en la PUCMM. ORCID ID: 0000-0002-7766-9111. Email: lauraenjo@gmail.com 